\title{
Preoperative airway management improved lung function rapidly in pediatric obstructive sleep apnea: A retrospective case series study
}

\author{
Yujuan Yang ${ }^{1}$, Yu Zhang ${ }^{1}$, Yanyan Yang ${ }^{1}$, Xiumei Chen ${ }^{1}$, Yakui Mou ${ }^{1}$, Liping Liu ${ }^{1}$, \\ Yuemei Sun ${ }^{1}$, Ningbo Tang ${ }^{1}$, and Xicheng Song ${ }^{1}$ \\ ${ }^{1}$ Qindao University Medical College Affiliated Yantai Yuhuangding Hospital
}

September 28, 2020

\begin{abstract}
Objectives: To establish an optimized airway management process to improve preoperative lung dysfunction in obstructive sleep apnea (OSA). Design: A retrospective case series study Setting: Tertiary university hospital in China Participants: The study included 483 children (319 males and 164 females; 6y to14y years) with OSA who underwent an adenotonsillectomy from November 2017 to December 2018. Main outcome measures: Children with OSA and who had abnormal airway function were identified by lung function test, and the risk factors for abnormal lung function were assessed. Next, the children received individualized atomization intervention based on the severity of their abnormal lung function, and the improvement in lung function was evaluated. Results: Lung function tests revealed that 45 patients had obstructive ventilation dysfunction, and histories of chronic cough or asthma were identified as risk factors for perioperative abnormal lung function. The FEV1\% pre exceeded $80 \%$ after 2 days of atomization intervention in 27 of 28 mild cases, 4 of 13 moderate cases, but in none of the 4 moderate-severe cases. After 4 days of atomization intervention, the FEV1\%pre of the remaining 14 patients in the three groups all increased up to $80 \%$. Other indicators of lung function (e.g., FEV1/FVC\% pre, MEF50\% pre, MEF $25 \%$ pre, and MMEF\% pre) were also greatly improved following the improvement of FEV1\% pre. No perioperative airway complications occurred. Conclusion: Prior to performing surgery on children with OSA and who have risk factors associated with abnormal lung function, it is potentially beneficial to establish an optimized airway management process to improve lung function before adenotonsillectomy. Keywords: Children, obstructive sleep apnea, airway management, lung function, surgical risk
\end{abstract}

\section{Introduction}

It has been reported that $6 \%$ of children with obstructive sleep apnea (OSA) experience perioperative complications. The overall respiratory complication rate was found to be $2.4 \%$ and the breakdown was as follows: laryngospasm/bronchospasm $(0.6 \%)$, pneumonia $(1.3 \%)$, postoperative lung edema $(0.6 \%)$, and other respiratory complications $(1.3 \%) \cdot{ }^{[1,2]}$ It is generally believed that the degree of preoperative pulmonary dysfunction has a significant influence on the incidence of perioperative pulmonary complications. Adenotonsillar hypertrophy is the main cause of pediatric OSA, and it can directly lead to a decline in lung function. ${ }^{[3]}$ When accompanied by allergic rhinitis, bronchial asthma, and other airway inflammatory diseases, the decrease of pulmonary function is more obvious. ${ }^{[4,5]}$ Abnormal lung function can objectively reflect airway hyperresponsiveness, which is associated with an increased risk for adverse airway events in children undergoing surgery. ${ }^{[6]}$ Furthermore, decreased lung function can increase the risk for lung infections caused by weakened respiratory movements and obstructed airway secretions after an adenotonsillectomy. ${ }^{[7]}$

Perioperative airway management, including preoperative use of positive pressure ventilation, and/or difficult intubation anesthesia implement mask ventilation, use of protective ventilation, morphine reserved strategy and half sitting position, and close monitoring after surgery, can reduce lung complications, patient mortality, readmission rates, and medical expenditures. ${ }^{[8-10]}$ The current preoperative airway assessment of OSA patients focuses on whether it is a difficult airway, and there are few reports on whether preoperative pulmonary 
function assessment and intervention can effectively reduce perioperative complications in OSA patients. The worse the preoperative pulmonary function, the more postoperative pulmonary complications. ${ }^{[11]}$ Therefore, it is beneficial to know how to avoid the risk for abnormal lung function so as to reduce the risk for airway complications associated with an adenotonsillectomy in children with OSA. This study was conducted to screen pediatric OSA patients with abnormal lung function and to establish an optimized preoperative airway management process that might promote a rapid improvement in lung function prior to surgery.

\section{Materials and Methods}

\section{Patients}

Patients or their parents who were unwilling to accept the airway management process or patients who were unable to tolerate the lung function test were excluded. Patients with restrictive ventilatory dysfunction (forced vital capacity $<80 \%$ predicted, forced expiratory volume in 1 second/forced vital capacity[?]0.70) were excluded because atomization therapy was not effective in improving restrictive ventilatory dysfunction. Finally, a total of 483 children with OSA (319 males and 164 females; age range, 6 y to 14 y) who underwent adenotonsillectomy were included in the retrospective study. All the children had a history of diverse symptoms associated with OSA. They received standardized polysomnography (PSG) after admission. An apnea hyponea index (AHI) [?] 2 events/hour or an obstructive apnea index (OAI)[?]1 event/hour was defined as a diagnostic criterion of OSA. ${ }^{[12]}$ Otolaryngologists evaluated the indication for adenoidectomy or tonsillectomy according to the degree of adenoid and tonsillar hypertrophy in children with OSA. ${ }^{[13,14]}$

\section{Clinical assessment}

Each patient completed a questionnaire and underwent a clinical examination. The questionnaire contained items concerning the patient's history of chronic cough, history of wheezing, familial and personal allergic history, history of asthma, as well as the subject's use of current medications. Eosinophil counts were determined by routine blood examinations and the reference value was $0.02-0.52 \times 10 / \mathrm{L}$. The fluoroenzyme immunoassay (UniCAP ${ }^{\circledR}$ 100, Pharmacia Diagnostics AB, Uppsala, Sweden) was used to measure inhaled allergen-specific IgE in serum. A positive test for specific IgE was defined as a value [?] $0.35 \mathrm{KU} / \mathrm{L}$. We defined obesity as a body mass index (BMI)[?] P95. ${ }^{[15]}$

\section{Lung function test}

A preoperative evaluation of lung function was performed, and the results were classified according to lung function test guidelines issued by the Chinese Society of Respiratory Medicine. ${ }^{[16]}$ Values were determined for the percentage of predicted value of the forced expiratory volume in the first second (FEV1\% pre), percentage of predicted value of the ratio of forced expiratory volume in the first second (FEV1) to forced vital capacity (FVC) (FEV1/FVC\% pre), percentage of predicted value of maximal mid-expiratory flow (MMEF\% pre), and the percentage of predicted value of maximal mid-expiratory flow mid-expiratory flow (MEF\% pre). The $\mathrm{FVC} \%$ pre is the main indicator of restrictive ventilatory dysfunction. The criterion for obstructive ventilation disorder was a FEV1/FVC $\%$ pre value [?] $92 \%$ or a FEV1\% pre $<80 \%$, and the criterion for small airway dysfunction was at least two values of MMEF\% pre, MEF50\% pre, and MEF $25 \%$ being lower than the lower limit of normal (LLN, 65\%). The severity of ventilatory dysfunction was based on FEV1\% pre: mild (70\%-79\%), moderate (60-69\%), moderate-severe (50\%-59\%), and severe (35\%-49\%), and very severe $(<35 \%)^{[17]}$

\section{Airway management process}

An otolaryngologist together with an allergist conducted a preoperative airway evaluation and then established an intervention process for each child with OSA based on their lung function test (Figure 1 ). In brief, we screened out patients based on their lung function test for that general anesthesia with tracheal intubation can elicit life-threatening bronchospasm in patients with bronchial hyperreactivity, and increased airway responsiveness is associated with an enhanced risk for respiratory symptoms and accelerated decline in FEV1. ${ }^{[18]}$ Therefore, an abnormal FEV1\% were considered as risk factor for airway complications. Patients with a FEV1\% pre value $<80 \%$ were given the atomized drugs at different frequencies according to 
the severity of their abnormal lung function. Atomization inhalation of $1 \mathrm{mg}$ budesonide, $0.25 \mathrm{mg}$ ipratropium, and $2.5 \mathrm{mg}$ terbutaline was given twice a day to mild patients and three times a day to moderate and moderate-severe patients. The exclusion criterion for preoperative airway risk was determined based on the patient's improvement in lung function after intervention.

\section{Statistical analysis}

All statistical calculations were performed using Graphpad Prism 7 software. The paired t test was used to evaluate the statistical significance of differences in lung function index before and after atomization. Categorical variables were compared using the Chi-squared test. Risk factors for abnormal lung function were analyzed by multivariate logistic regression, and the OR value and $95 \%$ confidence interval (CI) for each risk factor were calculated. P-values $<0.05$ was considered to be statistically significant.

\section{Results}

\section{Screening for abnormal lung function in children with OSA}

We evaluated the lung function in 483 children with OSA, and then divided the patients with abnormal lung function into mild, moderate and moderate-severe groups based on the severity of lung dysfunction (Table 1).

\section{Analysis of risk factors for abnor mal lung function in children with OSA}

The Chi-squared test was used to assess risk factors for abnormal lung function in children with OSA. Our results showed that the incidence of abnormal lung function among children with OSA was closely related to a previous history of chronic cough $\left(\chi^{2}=53.504, \mathrm{P}_{j} 0.001\right)$, wheezing $\left(\chi^{2}=40.136, \mathrm{P}_{j} 0.001\right)$, familial allergic diseases $\left(\chi^{2}=4.073, \mathrm{P}=0.044\right)$, and asthma $\left(\chi^{2}=197.700, \mathrm{P} j 0.001\right)$ (Table 2). A multivariate logistic analysis indicated that a previous history of chronic cough $\left(\mathrm{P}_{i} 0.001\right)$ or a previous history of asthma $\left(\mathrm{P}_{j} 0.001\right)$ were independent risk factors for abnormal lung function in children with OSA(Table 3) .

Improvement of preoperative abnormal lung function after individualized airway intervention

To further improve abnormal lung function to prevent airway complications, we treated these patients with individualized atomization intervention and tested lung function. Lung function tests showed that the FEV1\% pre values of the 45 patients were all increased up to $80 \%$ after atomization inhalation before surgery. Among them, the FEV1\% pre values of 27 mild patients and 4 moderate patients were increased up to $80 \%$ after 2 days of atomization intervention, and FEV1\%pre values of the remaining 1 mild patient, 9 moderate patients and 4 moderate-severe patients all reached $80 \%$ after 4 days of atomization intervention (Figure 2A, B) . In addition, we also found that the indexes of lung function (e.g., FEV1\%pre, FEV1/FVC\% pre, MEF50\% pre, MEF25\% pre, and MMEF\% pre) in each patient with mild, moderate or moderate-severe lung function obstruction were improved significantly after different frequencies of atomization inhalation(Figure 2C-F) .

\section{Incidence of high-risk perioperative airway events}

For patients with airway hyperresponsiveness, anesthetists took a series of measures based on assessment of preoperative airway assessment such as atomization inhalation, reduced extubation and intubation irritation, etc. Preoperative airway management continued after surgery until discharge. None of the children had acute attack of asthma and bronchial hyperactivity events during the perioperative period, and no pulmonary complications occurred after surgery.

\section{Discussion}

\section{Synopsis}

The continuity that exists between the upper and lower respiratory tracts means that inflammation in those regions is rarely localized. ${ }^{[19]}$ Inflammation or an obstruction in the upper airways of children with OSA can cause mucosal inflammation of the lower respiratory tract, and affect a child's lung function. ${ }^{[20,21]}$ 
Moreover, a meta-analysis of complications associated with adenotonsillectomy in children showed that the incidence of respiratory complications after a adenotonsillectomy in children with OSA was 5-fold higher than that in children without OSA. ${ }^{[22]}$ Therefore, it is potentially beneficial to establish a standardized airway management process to reduce the incidence of perioperative airway complications in children with OSA. As the beginning of perioperative airway management, a preoperative lung function test is important for assessing the risks associated with anesthesia and the likelihood of lung complications after surgery. ${ }^{[23]}$ In our study, we optimized some airway management measures and first developed an airway management process to improve preoperative lung function effectively. We found that nearly $10 \%$ of the children with OSA had abnormal lung function by lung function test. Individualized interventions were applied for these children and the results showed that FEV1\% pre was greater than $80 \%$ in all patients after 2 days or 4 days of atomization inhalation which was given at different frequencies based on the severity of obstruction. Meanwhile, histories of chronic cough and asthma were identified as risk factors for perioperative abnormal lung function. We should pay attention to the patients with these risk factors and intervene with them preventively as well, even if they had normal lung function.

\section{Risk factors for lung dysfunction}

A variety of risk factors are related to impaired lung function in children with OSA. ${ }^{[24,25]}$ However, the independent risk factors for lung function decline in pediatric OSA patients are unknown. We used 12 clinical indicators, including a patient history and lab test results in our risk factor analysis, and found that histories of chronic cough and concomitant asthma were the independent risk factors for lung dysfunction in children with OSA. Asthma and asthma-like symptoms, protracted bronchitis, and upper airway cough

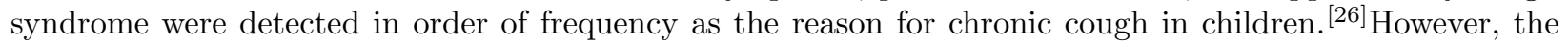
evidence for pediatric OSA as a cause of chronic cough is still ambiguous. An evaluation of children with chronic cough and obstructive sleep apnea at a single medical center showed that the superposition of both those factors on top of lower airway mucosal inflammation further damages lung function and aggravates the pre-existing airway hyper-responsiveness. ${ }^{[27]}$ Similarly, 31 of the 45 OSA patients with abnormal lung function in our study also had asthma. Although none of those patients had recently had an acute asthma attack, their poor lung function coupled with factors related to surgical stress caused us to believe that sedatives would greatly increase the risk for an acute attack of asthma during the perioperative period. ${ }^{[28,29]}$ Von Ungern-Sternberg et $\mathrm{al}^{[30]}$ demonstrated that an increased pediatric anesthetic risk for bronchospasm was closely associated with a positive respiratory history of nocturnal dry cough and wheezing, or a family history of asthma and atopy. In our study, wheezing and a family history of allergic diseases were associated with decreased lung function in the univariate analysis, but were meaningless in the multivariate logistic regression analysis. Wheezing is a common symptom of asthma, and asthma patients often have a family history of allergic diseases. ${ }^{[31]}$ We speculated that the collinearity between wheezing and a family history of allergic diseases or asthma led to that result. Therefore, when children with OSA have these risk factors for lung function decline (e.g., a history of chronic cough or concomitant asthma), special attention should be given to providing them with the follow-up airway intervention to help ensure the safety of surgery.

\section{Airway management process}

The clinical treatment of obstructive ventilation dysfunction is mainly designed to improve airflow and relieve smooth muscle spasms. Aerosolized inhalation, as the preferred drug delivery method for perioperative airway management in children, can effectively improve abnormal lung function. In our study, we provided combined atomization treatment with budesonide, ipratropium, and terbutaline to children with abnormal lung function. The combination of budesonide and terbutaline synergistically increases bronchodilatation and reduces airway hyper-responsiveness. Terbutaline can enhance hormone receptor sensitivity, and budesonide can promote the synthesis of $\beta 2$ receptors on the airway cell membrane. ${ }^{[32]}$ Ipratropium bromide, as a cholinergic receptor blocking drug, has a strong effect on bronchodilatation and can effectively inhibit airway inflammation and improve the lung ventilation ability of patients. The combination of three aerosolized drugs can rapidly improve lung function. ${ }^{[33]}$ We treated our patients with different atomization frequencies according to the severity of their obstructive ventilation dysfunction. An increase in the frequency of atom- 
ization exerts a greater superimposed effect on bronchial dilation on the basis of the last atomization, and makes it easier for drugs to enter the lower airway to relieve an airway obstruction. In our study, we treated mild patients with atomization twice a day and moderate or moderate-severe patients three times a day. Our results showed that all the FEV1\% pre improved up to $80 \%$ in these patients, irregardless of whether the patients had mild, moderate or moderate-severe airway obstruction. However, we needed to spend more time improving the FEV1\% pre in moderate-severe group compared with mild group and moderate group. Studies show that the inflammatory response of the respiratory mucosa and the neural reflex mechanism can induce bronchial constriction that increases airway hyper-reactivity in patients with moderate-severe OSA. ${ }^{[34]}$ Therefore, we needed to pay greater attention to such patients and employ adequate measures to improve their lung function. We also found that small airway dysfunction was present in $95.56 \%$ of patients with abnormal lung function. Studies have shown that OSA may be an independent risk factor for small airway diseases. ${ }^{[35]}$ Although there is no gold standard for diagnosing small airway disease, we still need attach importance to small airway dysfunction, because it is closely associated with poor asthma control, acute exacerbation of asthma, and the severity of airway hyper responsiveness. ${ }^{[36]}$ We found that small airway function indicators such as MEF50\% pre, MEF $25 \%$ pre, and MMEF\% pre, were all significantly improved after 2 days of atomization inhalation intervention.

\section{Conclusion}

Preoperative airway evaluation and intervention are the first step in perioperative airway management. In this study, we optimized some airway management measures, and further established a standardized preoperative airway management process for use in children with OSA, which showed it could rapidly improve abnormal lung function. In addition, patients with a history of chronic cough and asthma were more likely to have decreased lung function and could be further assessed/treated due to the potential increased risk for anesthesia. These results also suggest that for children who cannot complete lung function tests, if they have risk factors for lung dysfunction or symptoms of abnormal respiratory tract, we can give them preoperative atomization inhalation intervention to avoid possible perioperative airway adverse events. The disadvantage of this study was that there was no historical control group because previous children with OSA were not systematically examined for lung function before we established preoperative airway management procedures. However, the case series study can act as a pilot for future studies to provide a reference for preoperative standardized airway management.

\section{References}

[1] Kou YF, Sakai M, Shah GB, Mitchell RB, Johnson RF. Postoperative respiratory complications and racial disparities following inpatient pediatric tonsillectomy: A cross-sectional study. Laryngoscope. 2019;129(4):995-1000.

[2] De Luca Canto G, Pacheco-Pereira C, Aydinoz S, et al. Adenotonsillectomy Complications: A Metaanalysis. Pediatrics. 2015;136(4):702-718.

[3] Tapia IE, Marcus CL, McDonough JM, et al. Airway Resistance in Children with Obstructive Sleep Apnea Syndrome. Sleep. 2016;39(4):793-799.

[4] Oka S, Goto T, Hirayama A, Faridi MK, Camargo CA, Jr., Hasegawa K. Association of obstructive sleep apnea with severity of patients hospitalized for acute asthma. Ann Allergy Asthma Immunol. 2020;124(2):165-170.e4.

[5] Giraldo-Cadavid LF, Perdomo-Sanchez K, Cordoba-Gravini JL, et al. Allergic Rhinitis and OSA in Children Residing at a High Altitude. Chest. 2020;157(2):384-393.

[6] Ramgolam A, Hall GL, Zhang G, Hegarty M, von Ungern-Sternberg BS. Prediction of peri-operative adverse respiratory events in children: the role of exhaled nitric oxide. Anaesthesia. 2015;70(10):1160-1164.

[7] von Ungern-Sternberg BS, Ramgolam A, Hall GL, Sly PD, Habre W. Peri-operative adverse respiratory events in children. Anaesthesia. 2015;70(4):440-444. 
[8] Agostini P, Cieslik H, Rathinam S, et al. Postoperative lung complications following thoracic surgery: are there any modifiable risk factors? Thorax. 2010;65(9):815-818.

[9] Stock MC, Downs JB, Gauer PK, Alster JM, Imrey PB. Prevention of postoperative lung complications with CPAP, incentive spirometry, and conservative therapy. Chest. 1985;87(2):151-157.

[10] O'Gara B, Talmor D. Perioperative lung protective ventilation. BMJ. 2018;362:k3030.

[11] Takahashi Y, Suzuki S. Preoperative pulmonary function testing and postoperative complications. Journal of thoracic disease. 2018;10(Suppl 33):S3840-s3842.

[12] Paruthi S, Buchanan P, Weng J, et al. Effect of Adenotonsillectomy on Parent-Reported Sleepiness in Children with Obstructive Sleep Apnea. Sleep. 2016;39(11):2005-2012.

[13] Kuhle S, Hoffmann DU, Mitra S, Urschitz MS. Anti-inflammatory medications for obstructive sleep apnoea in children. Cochrane Database Syst Rev. 2020;1:Cd007074.

[14] Bixler EO, Vgontzas AN, Lin HM, et al. Sleep disordered breathing in children in a general population sample: prevalence and risk factors. Sleep. 2009;32(6):731-736.

[15] Ogden CL,Carroll MD,Kit BK,et al.Prevalence of Childhood and Adult Obesity in the United States, 2011-2012. JAMA.2014,311:806-814.

[16] Task Force for Lung Function, Chinese Thoracic Society. Series "Guideline for Lung Function Testing" Part2: Standardisation of spirometry. Zhonghua Jie He He Hu Xi Za Zhi.2014,37(7):481-486. DOI: 10.3760/cma.j.issn.1001-0939.2014.07.001.

[17] Quanjer PH, Pretto JJ, Brazzale DJ, et al. Grading the severity of airways obstruction: new wine in new bottles. Eur Respir J. 2014 Feb;43(2):505-512.

[18] Juusela M, Pallasaho P, Sarna S, Piirilä P, Lundbäck B, Sovijärvi A. Bronchial hyperresponsiveness in an adult population in Helsinki: decreased FEV1, the main determinant. Clin Respir J. 2013;7(1):34-44.

[19] Kanda A, Kobayashi Y, Asako M, Tomoda K, Kawauchi H, Iwai H. Regulation of Interaction between the Upper and Lower Airways in United Airway Disease. Med Sci (Basel). 2019;7(2).

[20] Marcus CL, Moore RH, Rosen CL, et al. A randomized trial of adenotonsillectomy for childhood sleep apnea. N Engl J Med. 2013;368(25):2366-2376.

[21] Verhulst SL, Aerts L, Jacobs S, et al. Sleep-disordered breathing, obesity, and airway inflammation in children and adolescents. Chest. 2008;134(6):1169-1175.

[22] De Luca Canto G, Pachêco-Pereira C, Aydinoz S, et al. Adenotonsillectomy Complications: A Metaanalysis. Pediatrics. 2015;136(4):702-718.

[23] Reddi BA, Johnston SD, Bart S, Chan JC, Finnis M. Abnormal lung function tests are associated with prolonged ventilation and risk of complications following elective cardiac surgery. Anaesth Intensive Care. 2019;47(6):510-515.

[24] Bui DS, Lodge CJ, Burgess JA, et al. Childhood predictors of lung function trajectories and future COPD risk: a prospective cohort study from the first to the sixth decade of life. Lancet Respir Med. 2018;6(7):535544.

[25] Chang AB, Bush A, Grimwood K. Bronchiectasis in children: diagnosis and treatment. Lancet. 2018;392(10150):866-879.

[26] Michaudet C, Malaty J. Chronic Cough: Evaluation and Management. Am Fam Physician. 2017;96(9):575-580. 
[27] Çolak Y, Afzal S, Lange P, Laursen LC, Nordestgaard BG, Dahl M. Role and Impact of Chronic Cough in Individuals with Asthma From the General Population. J Allergy Clin Immunol Pract. 2019;7(6):17831792.e1788.

[28] Michel F, Vacher T, Julien-Marsollier F, et al. Peri-operative respiratory adverse events in children with upper respiratory tract infections allowed to proceed with anaesthesia: A French national cohort study. Eur J Anaesthesiol. 2018;35(12):919-928.

[29] Woods BD, Sladen RN. Perioperative considerations for the patient with asthma and bronchospasm. Br J Anaesth. 2009;103 Suppl 1:i57-65.

[30] von Ungern-Sternberg BS, Boda K, Chambers NA, et al. Risk assessment for respiratory complications in paediatric anaesthesia: a prospective cohort study. Lancet. 2010;376(9743):773-783.

[31] Duijts L, Granell R, Sterne JA, Henderson AJ. Childhood wheezing phenotypes influence asthma, lung function and exhaled nitric oxide fraction in adolescence. Eur Respir J. 2016;47(2):510-519.

[32] Xiong G, Xu L, Wei L, Li X. Atomization inhalation of terbutaline and budesonide efficiently improved immunity and lung function of AECOPD patients. Cell Mol Immunol. 2008;5(4):287-291.

[33] Hancox RJ, Cowan JO, Flannery EM, et al. Randomised trial of an inhaled beta2 agonist, inhaled corticosteroid and their combination in the treatment of asthma. Thorax. 1999;54(6):482-487.

[34] Reddi BA, Johnston SD, Bart S, Chan JC, Finnis M. Abnormal lung function tests are associated with prolonged ventilation and risk of complications following elective cardiac surgery. Anaesth Intensive Care. 2019;47(6):510-515.

[35] Zerah-Lancner F, Lofaso F, Coste A, Ricolfi F, Goldenberg F, Harf A. Lung function in obese snorers with or without sleep apnea syndrome. Am J Respir Crit Care Med. 1997;156(2 Pt 1):522-527.

[36] Cottini M, Lombardi C, Micheletto C. Small airway dysfunction and bronchial asthma control : the state of the art. Asthma Res Pract. 2015;1:13.

Table 1. Lung function in 483 pediatric OSA patients

\begin{tabular}{lll}
\hline Variables & Cases (n) & Percentage (\%) \\
\hline Normal lung function & 438 & 90.683 \\
Obstructive ventilatory dysfunction & 45 & 9.317 \\
Mild & 28 & 6.393 \\
Moderate & 13 & 2.968 \\
Moderate-severe & 4 & 0.913 \\
\hline
\end{tabular}

Table 2. Clinical characteristics of 483 pediatric OSA patients

\begin{tabular}{|c|c|c|c|c|}
\hline Variables & Normal lung function (n) & Abnormal lung function (n) & $\chi^{2}$ & $\mathrm{P}$ \\
\hline Sex & & & 2.001 & 0.157 \\
\hline Male & 285 & 34 & & \\
\hline Female & 153 & 11 & & \\
\hline Obesity & & & 1.678 & 0.195 \\
\hline Yes & 125 & 17 & & \\
\hline No & 313 & 28 & & \\
\hline Inhaled allergen-specific IgE in serum & & & 1.853 & 0.173 \\
\hline Positive & 141 & 19 & & \\
\hline Negative & 297 & 26 & & \\
\hline Number of eosinophils in peripheral blood & & & 0.798 & 0.372 \\
\hline
\end{tabular}




\begin{tabular}{|c|c|c|c|c|}
\hline Variables & Normal lung function (n) & Abnormal lung function (n) & $\chi^{2}$ & $\mathrm{P}$ \\
\hline Abnormal & 17 & 3 & & \\
\hline Normal & 421 & 42 & & \\
\hline History of chronic cough & & & 53.504 & $¡ 0.001$ \\
\hline Yes & 81 & 30 & & \\
\hline No & 357 & 15 & & \\
\hline History of wheezing & & & 40.136 & $¡ 0.001$ \\
\hline Yes & 60 & 23 & & \\
\hline No & 378 & 22 & & \\
\hline History of familial allergic diseases & & & 4.073 & 0.044 \\
\hline Yes & 166 & 24 & & \\
\hline No & 272 & 21 & & \\
\hline History of personal allergic diseases & & & 0.147 & 0.702 \\
\hline Yes & 240 & 26 & & \\
\hline No & 198 & 19 & & \\
\hline Asthma & & & 197.700 & $¡ 0.001$ \\
\hline Yes & 16 & 31 & & \\
\hline No & 422 & 14 & & \\
\hline Allergic rhinitis & & & 0.205 & 0.651 \\
\hline Yes & 85 & 10 & & \\
\hline No & 353 & 35 & & \\
\hline
\end{tabular}

Table 3. Multivariate logistic analysis of risk factors in pediatric OSA patients with abnormal lung function

\begin{tabular}{llllll}
\hline Variables & S.E. & Wald & P & B & Exp (B) (95\%CI) \\
\hline History of chronic cough & 0.461 & 4.462 & 0.035 & -0.973 & $0.378(0.153-0.932)$ \\
Concomitant asthma & 0.549 & 45.052 & 0.000 & -3.682 & $0.025(0.009-0.074)$ \\
\hline
\end{tabular}

\section{Figures}

Figure 1. The airway management process involved in preoperative airway evaluation and the intervention performed for pediatric OSA patients.

Figure 2.Improvement of lung function indicators after atomization treatment.

- signifies that lung function index accounts for $80 \% ;-.-.-, 92 \% ; \ldots . ., 65 \%$. Mild, the mild group; Moderate, the moderate group; Moderate-severe, the moderate-severe group. 

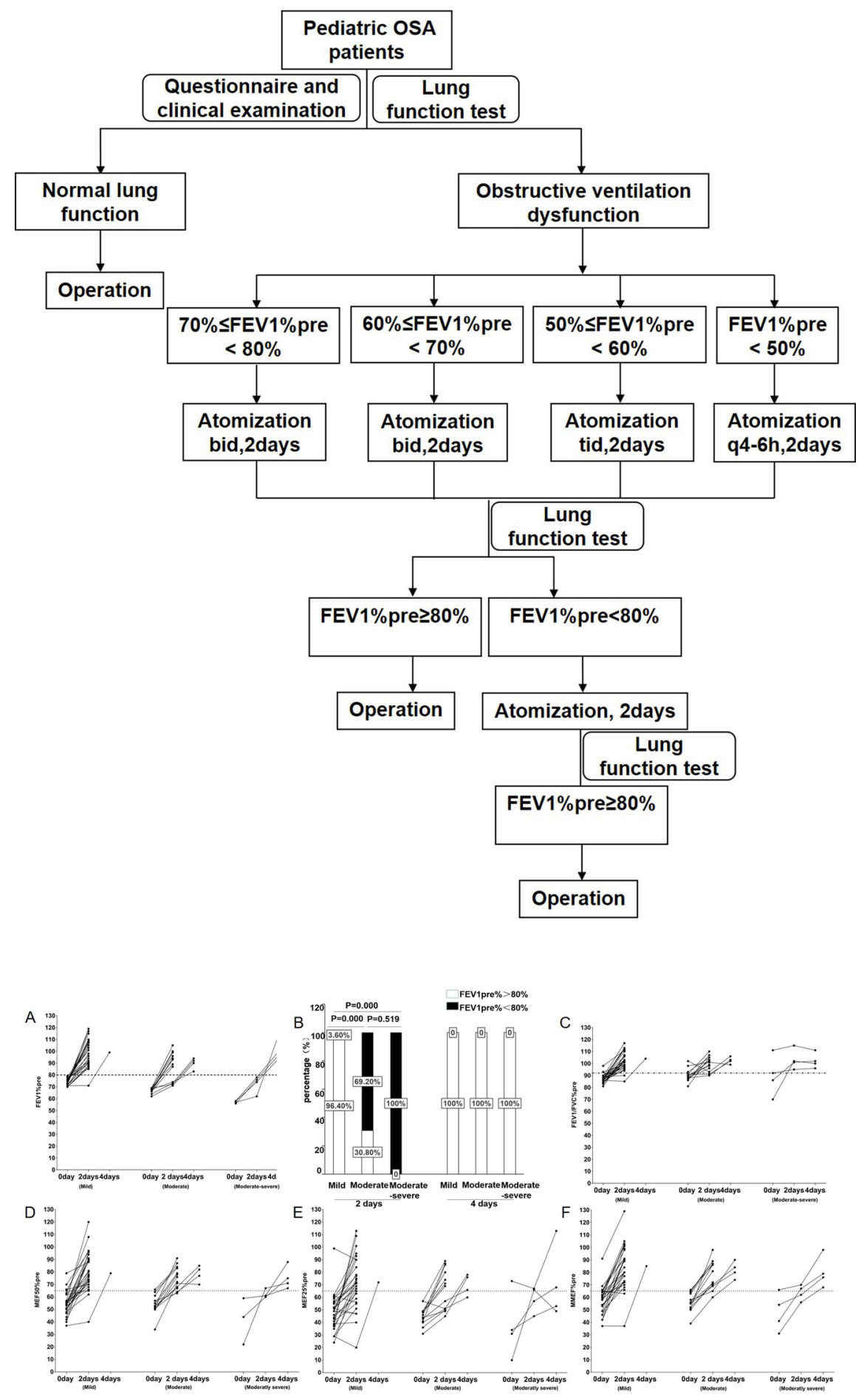